\title{
Emergent elemental two-dimensional materials beyond graphene
}

Yuanbo Zhang ${ }^{1}$, Angel Rubio ${ }^{2,3}$ and Guy Le Lay ${ }^{4}$

${ }^{1}$ State Key Laboratory of Surface Physics and Department of Physics, Fudan University, Shanghai 200433, China.

${ }^{2}$ Max Planck Institute for the Structure and Dynamics of Matter, and Center for FreeElectron Laser Science Luruper Chaussee 149, 22761 Hamburg, Germany.

${ }^{3}$ Nano-Bio Spectroscopy group, Universidad del Pais Vasco UPV/EHU, E-20018 San Sebastian, Spain.

${ }^{4}$ Aix-Marseille Université, CNRS, PIIM UMR 7345, 13397 Marseille, France.

Email: zhyb@ fudan.edu.cn ; angel.rubio@ mpsd.mpg.de ; guy.lelay@univ-amu.fr

\begin{abstract}
Two-dimensional (2D) materials may offer the ultimate scaling beyond the $5 \mathrm{~nm}$ gate length. The difficulty of reliably opening a band gap in graphene has led to the search for alternative, semiconducting 2D materials. Emerging classes of elemental 2D materials stand out for their compatibility with existing technologies and/or for their diverse, tunable electronic structures. Among this group, black phosphorene has recently shown superior semiconductor performances. Silicene and germanene feature Dirac-type band dispersions, much like graphene. Calculations show that most group IV and group V elements have one or more stable 2D allotropes, with properties potentially suitable for electronic and optoelectronic applications. Here, we review the advances in these fascinating elemental $2 \mathrm{D}$ materials and discuss progress and challenges in their applications in future opto- and nano-electronic devices.
\end{abstract}




\section{Introduction: The rise of elemental 2D electronic materials beyond graphene}

In the semiconductor sector, CMOS technology has played a key role in the last decades and helped follow Moore's law, which predicted that the number of transistors in a semiconductor chip roughly doubles every 18 months. Already, advanced CMOS miniaturization is at the "More Moore" domain, while "More than Moore" aims at progresses to enable non-digital micro/nanoelectronic functions. Novel disruptive routes must be explored to push the downscaling of the key electronic device, the field-effect transistor (FET) towards transistors with gate lengths of $5 \mathrm{~nm}$, typically to overcome the so-called ON/OFF and energy dissipation problems, i.e., detrimental short-channel effects. To this end, the use of 2D materials in the active channel of the transistor might be essential [1], and novel materials could be a source of innovation and performance gain [2].

The rise of 2D materials began in 2004 with graphene [3]. Since then, intensive research effort has made remarkable progresses in both basic science and electron-device technology of this material, as recently reviewed [4]. Digital devices requires an on-off ratio larger than $10^{4}$, and a good saturation of drain current is needed for power gain in a radio frequency (RF) FET [5]; graphene so far fares poorly on both counts due to the absence of a bandgap [6]. While researchers try to open an energy gap in graphene through various means (chemical funtionalisation [7], quantum confinement in nanoribbons [8], electric-field on bilayer [9] and tri-layer [10] graphene, AB sublattice symmetry breaking through substrate interaction [11], to name a few), another line of research pries the field wide open - the exploration of new functional 2D materials beyond graphene. Recent studies indicate that hundreds of 2D materials should exist [12]. Indeed, the experimentally accessible 2D materials grows at a rapid pace. Such a diverse family encompasses a large variety of material properties including insulators, semiconductors, semimetals and metals. An exciting aspect of 2D materials is to take advantage of the vastly different properties of different layered 
materials, and stack them into heterostructures to create novel devices made of atomically thin components [13]. Each 2D material known or to be discovered could thus serve as Lego brick to build new materials with tailored properties. In this context, elemental $2 \mathrm{D}$ materials [14] stand out for their simple composition/structure, but rich electronic properties that have raised huge interest and a cornucopia of emerging technological applications. Known elemental 2D materials are composed of III (B), group IV ( $\mathrm{Si}, \mathrm{Ge}, \mathrm{Sn}$ etc.) and group V (P, As etc.) elements, and so far borophene [15], silicene, germanene [16-18], stanene [19,20] and phosphorene have been shown to exist [21-24]. All these elemental 2D materials share similar buckled honeycomb-like lattices (see Fig.2), and yet their electronic structures differ significantly. Two additional advantages set elemental 2D materials apart from the rest of 2D materials. First, elemental 2D materials are inherently more compatible with existing semiconductor technologies than other 2D materials. Silicon and Germanium are backbones of traditional semiconductor industry, and group III and group V elements have long been used as dopants. Second, the mono-element composition may simplify the purification, growth and processing, and lead to high sample quality in the future.

In this review, we focus on the physics and properties of four elemental 2D materials: phosphorene, silicene, germanene and stanene, and will briefly touch on the others. Whenever possible, we will also assess the potential of making efficient FETs based on these 2D materials for electronic and optoelectronic applications based on what is known so far. In doing so, we note that the research into the elemental 2D FETs is still in its infancy (only recently the first field-effect transistors of few-layer phosphorene [25] and single-layer silicene [26] have been demonstrated). Even though remarkable progresses have been made in such a short period of time, the research has not yet come to the stage where comparison against the wish list of key figure of merits in traditional electron-devices [5] is meaningful. 
We will instead use such a wish list as a guide to pinpoint important outstanding problems in the field, and where research efforts should be directed next.

\section{The crystal structure of elemental 2D materials}

Let us start with carbon, whose ideal structure is a honeycomb lattice. The four valence electrons per carbon atom are exactly enough to fill all of the bonding electronic orbitals but none of the anti-bonding orbitals. Within the same group, germanium, silicon and tin have an electron configurations similar to carbon. Their most energetically favorable crystal structure is however the diamond structure. To obtain freestanding graphene, it is enough to come up with a smart procedure for isolating the weakly bound layers of graphite. The same is also true for other layered materials like hexagonal BN, black phosphorus, metal dichalcogenides and some oxides [13,27]. Si, Ge and $\mathrm{Sn}$, on the other hand, do not have a naturally occurring 3D-layered parent material. The synthesis of those freestanding 2D materials thus requires a more bottom-up approach, such as substrate mediated epitaxial growth.

The structure of germanene was first theoretically proposed together with that of silicene [16,17], while stanene was explored later [28-30]. In contrast to graphene, the honeycomb lattices of silicene and germanene are predicted to be buckled and composed of two vertically displaced sub-lattices with $\sim 0.4 \AA$ and $\sim 0.7 \AA$ buckling distances between the two A and B sublattices, respectively [17] (see Fig. 1). The larger ionic radius of Ge and Sn atoms make them even more in favor of $s p^{3}$ hybridization compared to silicon. This means that the $2 \mathrm{D}$ structures of $\mathrm{Ge}$ and $\mathrm{Sn}$ are even more vulnerable to 3D cluster formation than silicene. Yet, stabilization of germanene and stanene is possible on certain substrates as it is the case for silicene. To date, silicene, germanene and stanene have only been grown on metallic substrates ( $\mathrm{Si}$ and Ge, typically, on noble metal surfaces [21-23] and $\mathrm{Sn}$ on 
$\mathrm{Bi}_{2} \mathrm{Te}_{3}(111)$ [24], see Fig. 2). However, the recent experimental findings about the likely formation of hexagonal Si nanosheets on $\mathrm{MoS}_{2}$ could be quite promising [31].
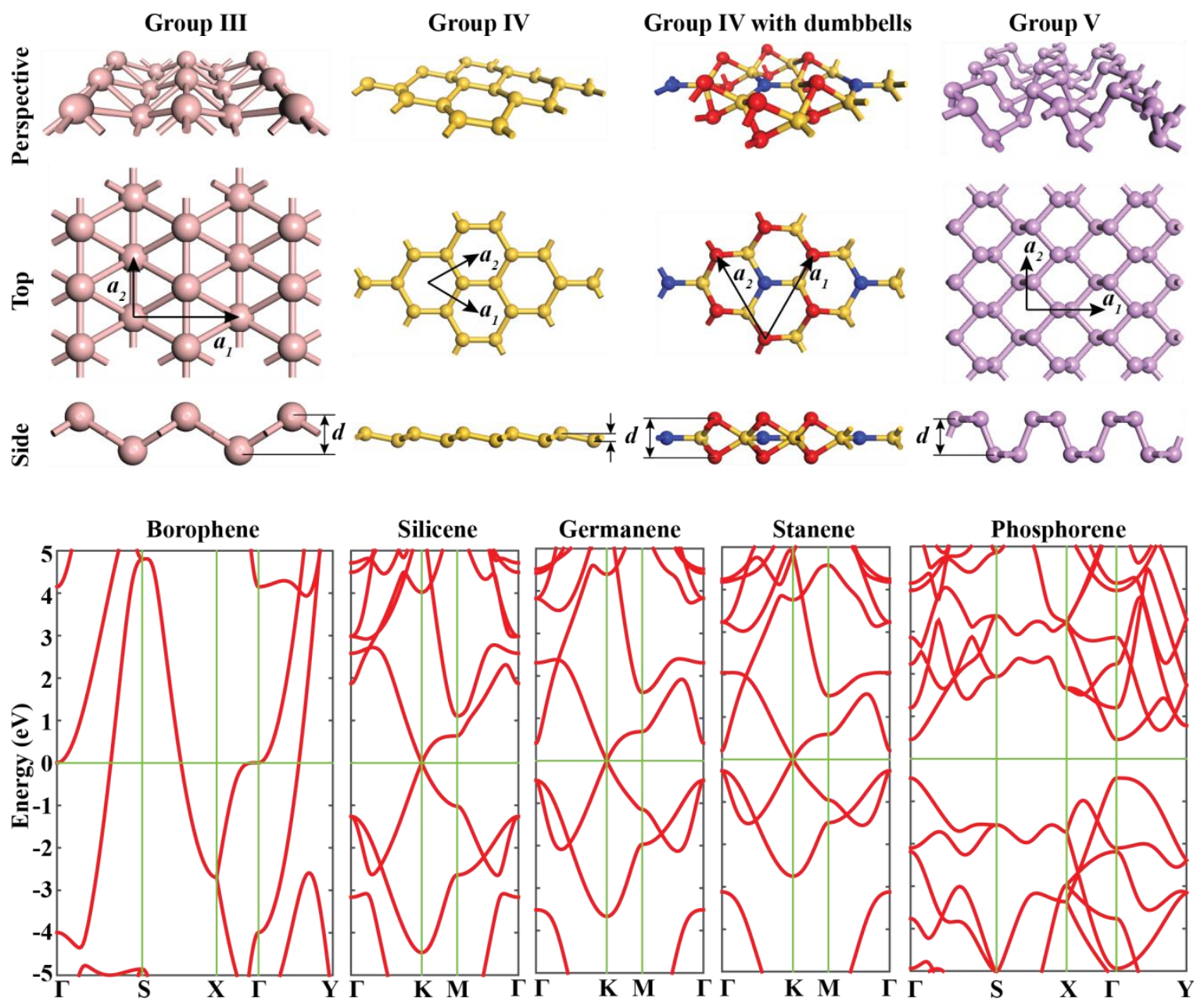

Figure 1. Crystal and electronic structures of group III, IV and V elemental 2D materials. Schematic atomic structures of the puckered honeycomb lattice of silicene, germanene and stanene. In contrast to the planar honeycomb lattice of graphene, the lattices are buckled by a distance $d$ in the out-of-plane direction. The lower panels display the calculated bandstructure of representative elemental 2D crystals in each group.

The archetype single layer silicene, which is the $3 \times 3$ phase having a unique orientation matching a $4 \times 4 \operatorname{Ag}(111)$ supercell, results from a delicate balance between the impinging $\mathrm{Si}$ flux, the surface diffusion of the deposited Si atoms on the bare and silicene covered areas and the competing in-diffusion toward the sub-surface. The growth is driven by these kinetic processes, which gives a very narrow substrate temperature window within about $200-220^{\circ} \mathrm{C}$. 
The Ag substrate plays a crucial role in growth of silicene. The "flower pattern" observed both in scanning tunneling microscopy (STM) and non-contact atomic force microscopy (AFM) imaging [21] results from the puckered Si atoms sitting nearly on top of Ag atoms, giving a total corrugation of $\sim 0.07 \mathrm{~nm}$ in the silicene sheet (see Fig. 2).

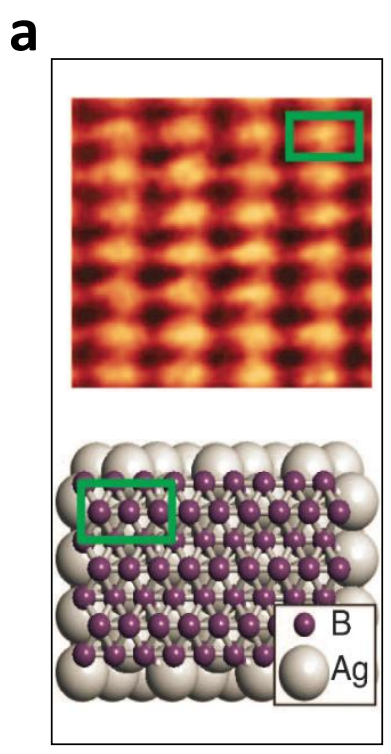

b

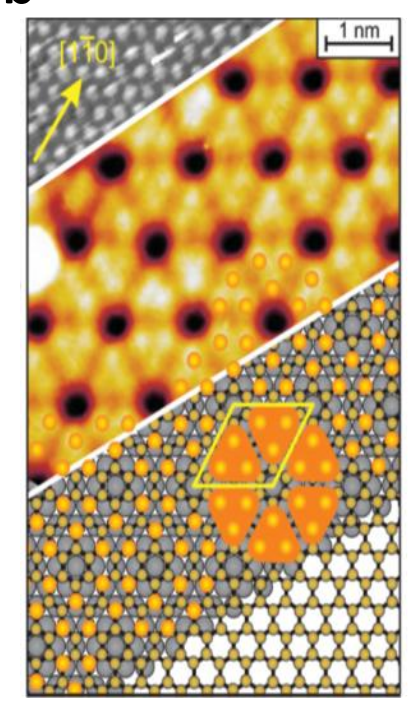

C
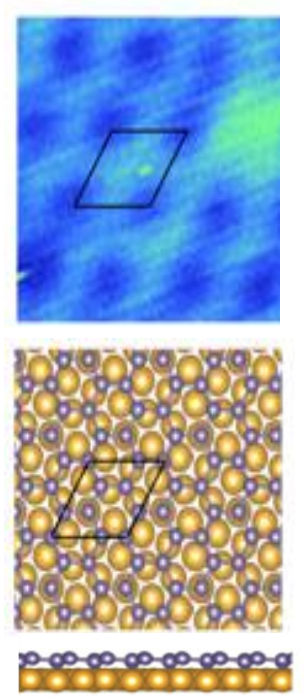

d
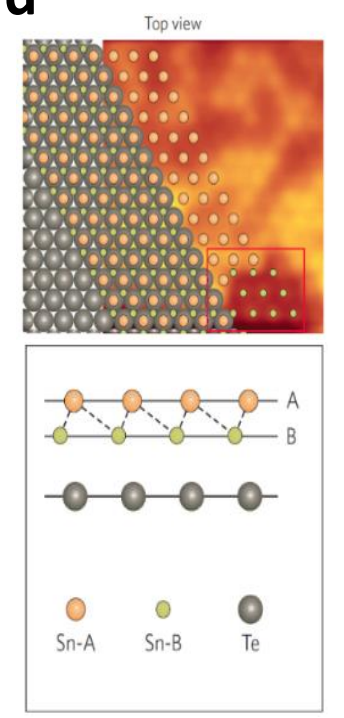

e
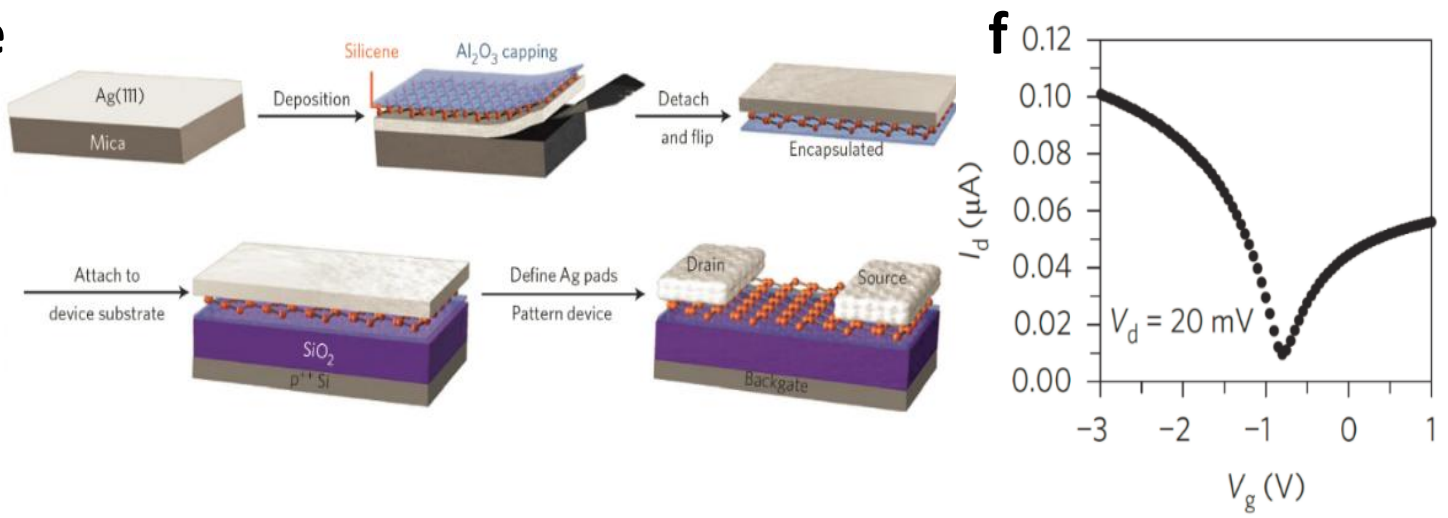

Figure 2. Monolayer silicene, germanene and stanene growth, and silicene field-effect transistor. a, STM image (upper panel) and atomic structure model of borophene grown on $\mathrm{Ag}(111)$ substrate. Adapted from ref. 15. b, Construction of the atomic structure model for 
the archetype $3 \times 3$ reconstructed silicene phase in coincidence with a $4 \times 4$ silver cell on $\mathrm{Ag}(111)$. Filled-states STM images of the initial clean $\mathrm{Ag}(111) 1 \times 1$ surface at the top left corner in grey and of the $3 \times 3$ silicene sheet in orange. Model of silicene on $\mathrm{Ag}(111)$ is shown below. Si atoms sitting on top of $\mathrm{Ag}$ atoms are highlighted as larger orange balls, resembling the measured STM image. In the bottom right corner, the ball-and-stick model for the freestanding silicene layer is shown with a Si-Si distance of $\sim 0.22 \mathrm{~nm}$. Adapted from ref. 21 . c, Experimental (upper panel) and simulated (middle panel) STM images of $\sqrt{3} \times \sqrt{3}$ reconstructed germanene grown on $\mathrm{Au}(111)$ according to the atomic model shown in the lower panel. Adapted from ref. 22 d, Top view of both the top and bottom Sn atoms of stanene on $\mathrm{Bi}_{2} \mathrm{Te}_{3}(111)$. The bottom $\mathrm{Sn}$ atoms were observed within the red rectangle. The atomic structure model (shown in the lower panel) has been superimposed on the STM image. Adapted from ref. 24. e, Schematic of the fabrication process of a back-gated FET with a silicene channel and with source/drain contacts defined in the native silver film. $\mathbf{f}$. The transfer characteristics of the silicene device exhibits an ambipolar behaviour. Adapted from ref. 32.

The study of silicene growth beyond monolayer reveals detailed reaction pathway. A Si adatom first attaches to silicene dangling bonds and then forms bridge bonds with two second neighbor $\mathrm{Si}$ atoms of silicene, thereby increasing their coordination number from three to four and forcing the atoms to move in the directions shown in Fig. 2b. The same happens to Ge and Sn where the dumbbell arrangement is the lowest energy structure for freestanding monolayers by a few hundreds of meV with respect to the hexagonal configuration. The dumbbell formation is exothermic and occurs spontaneously without barriers. When $\mathrm{Si}, \mathrm{Ge}$ and $\mathrm{Sn}$ are grown on a template, the energetic orders of the different structures may change by the interaction with the substrate. For the case of $\mathrm{Si}$ grown on a $\mathrm{Ag}(111)$ surface, the monolayer honeycomb structure has the lowest formation energy. It is also the case for Ge grown on an $\mathrm{Au}(111)$ surface [22]. Theoretically, one of the authors (A.R.) has proposed that after growth of the silicene monolayer honeycomb structure $(3 \times 3$ on $4 \times 4 \mathrm{Ag}(111))$, the dumbbell structure corresponding to a $\sqrt{3} \times \sqrt{3}$ phase will naturally form explaining the experimental observations [33]. There are however alternative explanations for this $\sqrt{3} \times \sqrt{3}$ phase in terms of either a weak surface honeycomb reconstruction [34] or a Si-Ag surface alloy structure on top of ultra-thin diamond-like $\mathrm{Si}(111)$ [35]. 
Looking now to the neighboring columns in the periodic table we have boron and phosphorous as potential candidates to form $2 \mathrm{D}$ materials due to their rich chemistry. However in contrast to $\mathrm{P}$ the hexagonal boron sheet is theoretically predicted to be unstable [36]. Boron has been shown to have metallic layered structure composed of buckled triangular motifs in a partially filled honeycomb structure at a specific hole density $[36,37]$. This configuration allows the kind of chemical bonds that boron forms best: bonds among three atoms rather than between two. However, as for silicene, germanene and stanene, a hexagonal boron layer could be stabilized by the interaction with a substrate. Indeed crystalline $2 \mathrm{D}$ boron sheets (i.e., borophene) have been recently synthesized on silver surfaces [15]. On the other hand, bulk black phosphorous appears in three crystalline structures: orthorhombic, simple cubic, and rhombohedral. The single layer black phosphorous includes two atomic planes and two kinds of P-P bonds: the shorter one connects nearest $\mathrm{P}$ atoms in the same plane, and the longer one connects $\mathrm{P}$ atoms between the top and bottom of the layer (Fig. 1). The thickness of the phosphorene layer is $2.10 \AA$. Although the threefold coordination of $\mathrm{P}$ atoms in phosphorene resembles the honeycomb structure of silicene, there are significant differences. The phosphorene structure is reminiscent of the (110) plane of the diamond structure while silicene is related to the (111) plane. The bond angles of phosphorene are significantly smaller than the tetrahedral angle. This anisotropic structure is at the origin of some of its interesting electronic and mechanical properties, i.e., flexibility under tensile strain, giant thermoelectric coefficient, such that optoelectronic devices in the mid- and near-infrared spectrum ranges are envisaged, typically, detectors, modulators, and, potentially, light generation devices like light-emitting diodes and lasers, for high-speed flexible electronics systems operating in the multi GHz frequency range and beyond.

\section{Electronic structure of elemental 2D materials}


Free standing silicene, germanene and stanene share several peculiar electronic properties. Group IV 2D crystals have half-filled bands derived from $p_{z}$ orbitals which, under inversion symmetry, give Dirac points at symmetry points of the Brillouin zone that are robust against the buckling of the lattice. In graphene the electronic states of graphene near the Dirac ( $K$ and $\mathrm{K}^{\prime}$ ) points are described by a linear dispersion relation with a Fermi velocity on the order of $10^{6} \mathrm{~m} / \mathrm{s}$. A similar linear dispersion was observed in $3 \times 3$ and $\sqrt{3} \times \sqrt{3}$ silicene $[21,38]$ by angle-resolved photoemission spectroscopy (ARPES) measurements and by analyzing the quasi-particle interference pattern captured by STM measurements [39] (but not on the isolated silver surface). Yet, the band structure of silicene is significantly modified, not only by the $3 \times 3$ reconstruction but also by the strong hybridization with the Ag substrate $[33,40]$ (therefore the observed linear dispersion does not come from the Dirac bands of the isolated silicene monolayer).

Spin-orbit coupling (SOC) can open a band gap at the otherwise gapless Dirac points: $73.5 \mathrm{meV}, 23.9 \mathrm{meV}$ and $1.55 \mathrm{meV}$ gaps for stanene, germanene and silicene, respectively (note that the already weak spin-orbit coupling in graphene is even further suppressed by its flatness). These bulk gaps potentially support topologically protected gapless helical edge modes, which may lead to the quantum spin Hall effect (QSHE). In graphene the spin-orbit coupling is so weak that the QSHE can only occur at unrealistically low temperatures. Silicene, on the other hand, was shown to preserve its topologically nontrivial electronic structure despite buckling and can host QSHE up to $18 \mathrm{~K}$ [41]. The QSHE may occur at temperatures up to $277 \mathrm{~K}$ or higher in germanene and stanene [19,29], and the temperature could go even higher after functionalization [19]. Moreover, silicene, germanene, and stanene exhibit peculiar topological properties [42], such as topological superconductivity [41] and quantum anomalous Hall insulator upon photo-irradiation. Upon introducing $s$-wave superconductivity by proximity coupling, the system becomes a photo-induced chiral 
topological superconductor. Such topological superconductors possess Majorana bound states in vortex cores in two dimensions (or at the ends of one-dimensional structures), and the Majorana fermions exhibit non-Abelian exchange statistics which may be relevant for topological quantum computing [43].

The lack of a large bandgap may seem to be a limiting factor for the potential application of group IV 2D materials in nanoelectronics, as a bandgap of $\sim 0.4 \mathrm{eV}$ or more is needed for logic operations [5]. Band-gap engineering may mitigate this problem: band gap may be switched on and off by strain [44]; alternatively, an external electric field applied in a direction perpendicular to the buckled lattice can induce charge imbalance between the sublattices, and open a band gap [44]. In addition, a variable perpendicular electric field can induce a phase transition from a topological insulator to a trivial band insulator, passing through a valley-spin polarized metal [45], and can also induce superconducting states when doped. A tunable quantum phase transition from a singlet chiral $d+i d$ '-wave pairing phase to a triplet $f$-wave phase could occur, provoking an increase in the superconducting critical temperature [46]. This intriguing triplet superconducting state has time-reversal-invariant weak topological property and can harbor the Majorana zero-mode at its boundary [47].

Hydrogenation is another way of opening the band gap as demonstrated in hydrogenated graphene (the so called graphane) [48]. Graphane has been predicted to be a wide band-gap insulator with gap of $5.4 \mathrm{eV}$ and its optical properties are dominated by localized charge-transfer excitations stemming from the enhanced 2D screening [49]. The strong electron-hole interaction also leads to the appearance of small radius bound excitons that open the path towards the possibility of excitonic Bose-Einstein condensation [49]. Meanwhile, silicane and germanane are predicted to be stable in freestanding form being semiconductors with indirect $(\sim 3 \mathrm{eV})$ and direct $(\sim 2 \mathrm{eV})$ band gaps, respectively [50]. Fully hydrogenated group-IV monolayers are predicted to be nonmagnetic semiconductors. 
However, partially hydrogenated silicene, germanene with chair configuration may exhibit magnetic properties [51]. Varying H-concentration may also allow for the modulation of electronic properties for applications in nanoelectronic and nanophotonic devices [52].

Phosphorene, on the other hand, is known to be a semiconductor in its pristine form [53-55]. This reflects the fact that elements in group V have one more valence electron than those in group IV, so that the half-filled $\pi$ bands of the group IV 2D crystals are now fully filled. Although this simplified picture does not capture the full complexity of the electronic structure, calculations show that the states near the band edge are indeed $p_{z}$ in nature $[56,57]$. Direct measurement of the band gap in monolayer phosphorene is not yet available, and calculations [56,57] have put the value at $\sim 1.8 \mathrm{eV}$. As monolayers are stacked together to form few-layer crystal, the interlayer interaction gives rise to dispersive bands in the out-ofplane direction: a recurring theme in the 2D crystal family. In few-layer phosphorene, stacking has the effect of reducing the bandgap, from $\sim 1.8 \mathrm{eV}$ (in monolayer) to $\sim 0.3 \mathrm{eV}$ (in the bulk) [57-63]. The band gaps are direct so that the transition across the gap couples strongly to light. So by varying the number of layers (i.e. thickness), it is possible to tune the bandgap to cover visible red to mid-infrared (Fig. 3) - a wide spectral range that is important to solar energy harvesting, communication, and remote sensing. 

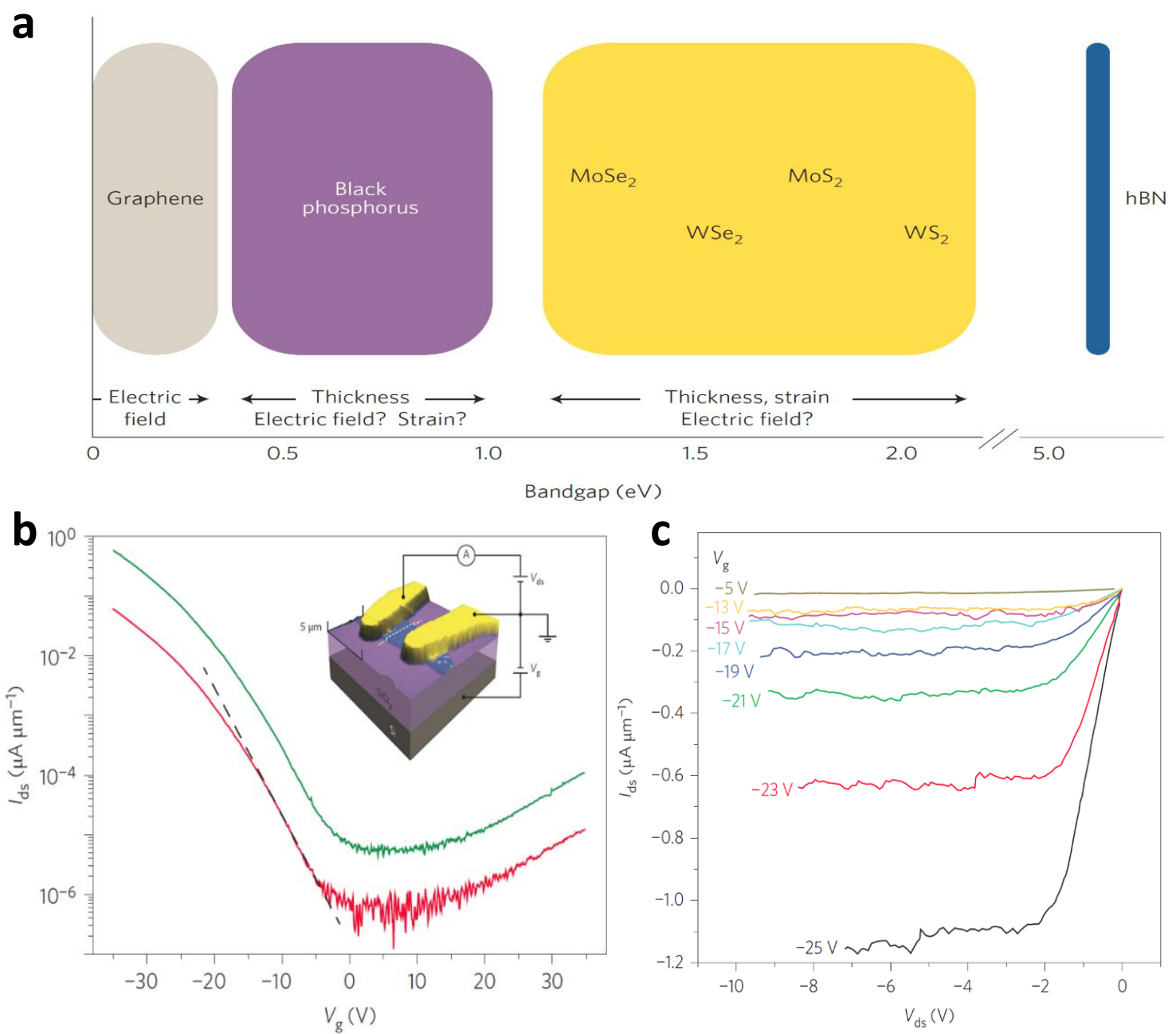

Figure 3. Bandgap of 2D materials and black phosphorus field-effect transistors. a. Bandgap energies of elemental 2D materials compared to several other layered materials used for nanoelectronics. The range of values for each material indicate the expected variation induced by a variety of means such as film thickness, electric field, or strain. Adapted from ref. 100. b, Source-drain current (on a logarithmic scale) as a function of gate voltage obtained from a black phosphorus FET with a 5-nm-thick channel with drain-source voltages of $10 \mathrm{mV}$ (red curve) and $100 \mathrm{mV}$ (green curve). Device was fabricated on a silicon substrate with $90 \mathrm{~nm} \mathrm{SiO}$. Channel length and width of the device are $1.6 \mathrm{~mm}$ and $4.8 \mathrm{~mm}$, respectively. Drain current modulation up to $\sim 10^{5}$ is observed for both drain-source biases on the hole side of the gate doping. Inset: Schematic of device structure of a few-layer phosphorene FET. Adapted from ref. 25. c, Drain-source current $I_{d s}$ as a function of bias $V_{d s}$ at different gate voltages showing the well-developed saturation behaviour of few-layer phosphorene FET. Adapted from ref. 25 
Few-layer phosphorene therefore has the potential to serve as a high performance material in optoelectronic devices. Moreover, the puckered orthorhombic structure induces strong in-plane anisotropy in its electronic, optical and phonon properties [64,65]. As a result, the effective mass of carriers along the zigzag direction is about ten times larger than that along the armchair direction [64]. The anisotropy also leads to a plasma resonance that has a vectorial dependence on the momentum, so that the plasma frequency can be tuned by the linear polarization direction of the incident light [66].

The fact that 2D crystal itself is a surface leads to a key advantage: it is strongly influenced by its environment so that external perturbations could be utilized to modulate its material properties. In particular, because all elemental 2D materials are buckled excepted for graphene, an electric field applied perpendicular to the sample plane could induce a potential difference on lattice sites above and below the plane that are otherwise symmetric. As a result, the electronic structure of the 2D materials is modulated by the electric field through the Stark effect. It has been predicted that silicene undergoes a topological phase transition as a function of the applied electrical field from a $Z_{2}$ topological insulator to a normal insulator in a strong enough electric field [45]. A similar effect is expected for germanene and stanene but with a required larger external electric field. In phosphorene, the electric field has the opposite effect of closing the band gap and reopens it to form a topological insulator [67]. The fieldinduced band closing has recently been observed on the surface of black phosphorus under potassium doping [68]. The tunable phase transition, if realized, could lead to the QSHE and opens the possibility of "field effect topological transistor" where both spins and charge can be manipulated simultaneously [67].

Pressure and strain has similar effect of closing the bandgap in black phosphorus [6975]. Although the covalent bond between phosphorus atoms is stiff against compression, a change in the bond angle in the puckered lattice allows for a large deformation in the armchair 
direction under a moderate pressure so black phosphorus is extremely soft. Because the direct band gap is mainly determined by the out-of-plane $p_{z}$-like orbital, a slight change in the bond angle of the puckered phosphorene layers strongly modulates the direct band gap. It has been shown experimentally that a moderate pressure $(\sim 1.2 \mathrm{GPa})$ induces an electronic transition from semiconductor into semimetal in bulk black phosphorus [75]. The semimetal phase is accompanied by a colossal magnetoresistance and the emergence of Dirac quasi-particles with non-trivial Berry phase [75]. Higher pressure (> $4 \mathrm{GPa}$ ) drives a structural phase transition from the orthorhombic to the rhombohedral phase, and the bulk black phosphorus turns into a metal (and a superconductor at low temperatures) [76,77]. So far such experimental investigations on effects of pressure/strain have been performed on bulk black phosphorus crystals. It remains to be seen whether mono- or few-layer phosphorene exhibits the electronic and structural transitions and hosts Dirac quasiparticles and superconductivity in the 2D limit when subjected to pressure/strain.

\section{Fabrication of elemental 2D materials}

The seminal paper on silicene growth reported a "flower pattern" observed in STM [21], which was later confirmed [78]. Remarkably, reflection high energy positron diffraction experiments gave the same atomic geometry of the $3 \times 3$ reconstructed silicene as the one derived from the DFT-GGA calculations [79], which was further confirmed in a dynamic low-energy electron diffraction (LEED) study [80]. Another 2D phase, formed at a slightly higher temperature with, again, no direct evidence of a honeycomb arrangement of the $\mathrm{Si}$ atoms in STM or AFM images, was understood in a similar fashion. The phase was interpreted as a rotated and slightly stretched $\sqrt{ } 7 \times \sqrt{ } 7 \mathrm{R}\left( \pm 19.1^{\circ}\right)$ silicene phase in coincidence with $\sqrt{ } 13 \times \sqrt{ } 3 \mathrm{R}\left( \pm 13.9^{\circ}\right)$ silver (111) supercells, giving 4 rotated (but symmetry equivalent) domains [81,82]. Finally, a pseudo “ $2 \sqrt{ } 3 \times 2 \sqrt{ } 3) \mathrm{R}\left( \pm 30^{\circ}\right)$ ” phase (in 
terms of $\mathrm{Ag}(111)$ basis vectors) prepared at significantly higher substrate temperatures $(\geq$ $270^{\circ} \mathrm{C}$ ) was shown to be a dying phase [83], which collapse into a surface alloy [83] at such elevated temperatures. Similar experimental evidence for buckled silicene on diboride thin films [84] and $\operatorname{Ir}(111)$ [85] was presented. So far the primitive monolayer silicene lattice has not been observed on any of these surfaces, because silicene invariably interacts with the substrates [86,87], typically forming hybridized states as demonstrated on $\mathrm{Ag}(111)$ [40]. Such hybridization also explains why no Landau levels were detected in tunneling spectroscopy measurements in high magnetic fields [88].

The success in synthesizing silicene on metallic templates has triggered research on germanene on analogous substrates. The first report in 2014 for buckled germanene on $\operatorname{Pt}(111)$ [89] was, however, questioned as surface alloy formation [90]. Also in 2014, a germanene phase on $\mathrm{Au}(111)$ surface (possibly incorporating few Au atoms) was discovered [22] among other phases that were not yet fully understood. The phase was identified as the nearly flat $\sqrt{ }$ $3 \times \sqrt{ } 3 \mathrm{R}\left(3^{\circ}\right)$ reconstructed germanene layer, in conjunction with a $\sqrt{ } 7 \times \sqrt{ } 7 \mathrm{R}\left(19.1^{\circ}\right)$ $\mathrm{Au}(111)$ supercell [22]. A significant progress was soon achieved [23] by depositing the Ge on an $\mathrm{Al}(111)$ template at only $80^{\circ} \mathrm{C}$. The process yields a unique phase that was recognized as continuous, uniform $2 \times 2$ reconstructed germanene in conjunction with $3 \times 3 \mathrm{Al}(111)$ supercell. This is very encouraging because such a pure phase will greatly facilitate detailed characterization and its transfer to an insulating substrate for device fabrication.

While the bottom-up growth of phosphorene has not yet been realized, top-down isolation of phosphorene thin flakes, both mechanically [25,91-94] and chemically [95-97], from bulk black phosphorus crystals has been successfully demonstrated. Both top-down approaches were developed in the early days of graphene research, and each of the two approaches provides distinct advantages. Mechanically exfoliated black phosphorus offers superior sample quality that is important for exploration of new physics in the clean limit, and 
for the development of proof-of-principle electronic/optoelectronic device prototypes, whereas chemical exfoliation is able to produce large quantity of the material for applications that do not need high quality. The availability of high quality, mechanically exfoliated fewlayer phosphorene has fueled the rapid progress in the understanding of its material properties and in the development of prototypical electronic and opto-electronic devices, as we shall discuss later.

\section{Elemental 2D field effect transistors}

Before 2014, a widely spread opinion, as expressed in ref. 13, is that "The $2 \mathrm{D}$ silicon can be grown epitaxially and investigated in high vacuum. However it is not expected to survive isolation from its parent substrate or exposure to air. With contemporary technologies, the silicene transistors envisaged in the literature cannot be made". More recently, the question "Can a silicene transistor be realized?" has been posed [98]. Hence, the announcement in 2014 of the realization of silicene FET operating at room temperature was a big surprise [26], and the ingenious fabrication procedure may be applicable to germanene and stanene as well. The authors grew single layer silicene under ultra-high vacuum on a thin epitaxial $\operatorname{Ag}(111)$ film on mica, encapsulated it in situ with a thin alumina film, and then delaminated the Ag-silicene-alumina sandwich in air and flipped it down onto a $\mathrm{SiO}_{2} / \mathrm{Si}$ wafer. Using wet etch, they could pattern the silicene channel and the source/drain electrodes, and at the same time retard the oxidation/degradation of the air-exposed silicon channel. Even though the silicene FET could only survive for a few minutes under ambient conditions, the authors managed to show an ambipolar charge transport with mobilities of $\sim 100 \mathrm{~cm}^{2} \mathrm{~V}^{-1} \mathrm{~s}^{-1}$ for both electron and holes and an ON/OFF ratio of about 10. This was a real success for a first try, with plenty of room for better performance in the future. 
The FETs based on few-layer phosphorene were first reported in 2013 (ref. 99), followed by several published works shortly after [25,91-94]. As black phosphorus has a bandgap of $\sim 0.3 \mathrm{eV}$, the FETs have a much improved switching performance with an on-off ratio up to $10^{5}$ (compared with $\sim 10-100$ in FETs based on gapless graphene and silicene). Thanks to the gap, well-behaved drain current saturation in the I-V characteristics has been demonstrated (Fig. 3c). Such saturation is important not only in digital transistors but also for improving power gain in RF analog devices [6]. It is important to note that so far black phosphorus flakes that are used to build the FET prototypes are at least a few nanometer thick. The bandgap in those flakes is still mostly in the bulk limit (i.e. $\sim 0.3 \mathrm{eV}$ ). The on-off ratio will certainly increase when monolayer phosphorene, which has a bandgap of $\sim 1.8 \mathrm{eV}$, is used in the future.

The quality of an electronic material is characterized by its carrier mobility, which largely determines how fast the device could operate in high-speed logic and RF electronics. High carrier mobilities are also crucial for studying the quantum transport in black phosphorus in the clean limit. Two types of mobilities are often reported in the literature: field-effect mobility and Hall mobility. The field-effect mobility is however prone to artificial inflation [101] so here we only refer to Hall mobility in our discussion. The first few-layer phosphorene FETs used $\mathrm{SiO}_{2}$ as the substrate, and the fabrication was done in air. Even though these are not conditions conducive to high mobility $\left(\mathrm{SiO}_{2}\right.$ substrates tend to have charged impurities and black phosphorus surfaces are known to degrade in air [102-105]), the FETs exhibit mobilities up to $1000 \mathrm{~cm}^{2} \mathrm{~V}^{-1} \mathrm{~s}^{-1}$ at room temperature. From the temperature dependence of the mobility (Fig. 4a), which follows that of typical semiconductors, it is clear that the room-temperature mobility is limited by phonon scattering, whereas the lowtemperature mobility is limited by impurities. 

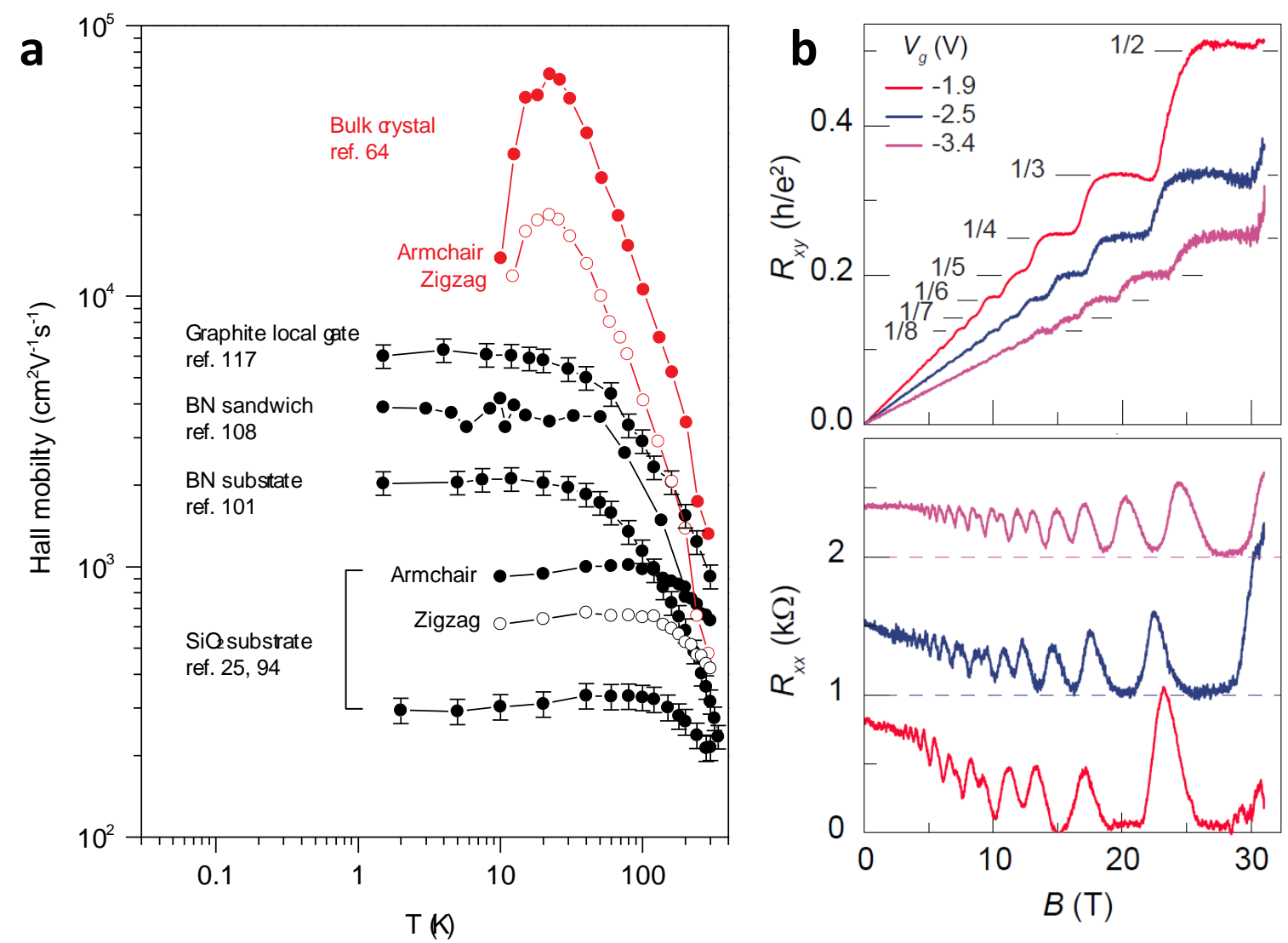

Figure 4. Mobility of black phosphorus FET and quantum Hall effect in black phosphorus 2D electron system. a, The mobility progress achieved for few-layer phosphorene FET over the last two years. The steps leading to the improvements are indicated. The curve labelled 'bulk' is for black phosphorus bulk crystal. "BN sandwich", black phosphorus thin film sandwiched between two hBN flakes; "Graphite local gate", black phosphorus 2DES gated by a graphite gate in close vicinity, which screens the impurities in the 2DES. "Armchair" and "Zigzag" label the mobilities measured in the armchair and zigzag directions of black phosphorus crystal, respectively. b, The quantum Hall effect in a black phosphorus 2D hole gas. Horizontal lines (upper panel) mark the quantized in the Hall resistance at $h / v e^{2}$, where $v$ is the integral filling factor. Adapted from ref. 117.

Much like the 2D electron systems (2DES) found in Si MOSFETs and GaAs/AlGaAs heterostructures [106], the 2D conductive channel of a black phosphorus FET is tightly confined to the surface layers by the gate electric field, and forms a new type of 2DES [101,107-109]. In the past two years, the carrier mobility of black phosphorus 2DES has improved tremendously (Fig. 4a), at a rapid pace seen in the early days of GaAs/AlGaAs heterostructure development [110]. The main task here is to reduce the effect of impurity 
scattering. To this end, the fabrication of high-mobility black phosphorus FETs has greatly benefitted from techniques developed in graphene research [111-116]. In particular, the lowtemperature mobility has gone up by one order of magnitude due primarily to two major improvements. First, most of the fabrication procedures are now moved into glove box where the $\mathrm{O}_{2}$ and water vapor content is kept low to mitigate sample surface degradation. Second, switching from $\mathrm{SiO}_{2}$ to $\mathrm{hBN}$ substrate reduces charged impurities at the interface. Both methods drastically decrease the impurity scattering. Further improvement was achieved by placing a graphite back gate very close to the conducting channel of the FET so that the electrons in metallic graphite could screen the charged impurities in the channel [117]. With all the advancement, the current record mobility now stands at $\sim 6000 \mathrm{~cm}^{2} \mathrm{~V}^{-1} \mathrm{~s}^{-1}$ for $2 \mathrm{D}$ electron gas (2DEG) and $~ 20006000 \mathrm{~cm}^{2} \mathrm{~V}^{-1} \mathrm{~s}^{-1}$ for 2D hole gas (2DHG) at low temperatures, whereas the phonon-limited room-temperature mobility only sees moderate improvement ( $1000 \mathrm{~cm}^{2} \mathrm{~V}^{-1} \mathrm{~s}^{-1}$; ref. 117). Further improvement of device mobility should be possible, as the bulk black phosphorus was shown to exhibit mobilities up to $70,000 \mathrm{~cm}^{2} \mathrm{~V}^{-1} \mathrm{~s}^{-1}$ (ref. 118).

The persistent mobility improvement culminated in the observation of quantum Hall effect (QHE) in high quality black phosphorus 2DES [117]. Before the QHE sets in, the formation of Landau levels in black phosphorus 2DEG leads to well-developed Shubnikov-de Haas $(\mathrm{SdH})$ oscillations, which provide a useful tool for probing Landau level energetics in black phosphorus 2DES. It has been shown that the spin-splitting energy in black phosphorus 2DES is an appreciable fraction of the cyclotron energy (up to $\sim 0.8$ for hole carriers) [117]. This is in stark contrast with graphene or GaAs-based 2DEGs, where the ratio of the two energy scales is of the order of $10^{-2}$, but rather close to $\mathrm{ZnO}$ system with a ratio of 0.95 [119]. In light of recent observations of peculiar even-denominator fractional $\mathrm{QH}$ states in $\mathrm{ZnO}$ based 2DES as a result of spin-splitting induced Landau level crossing [119], black 
phosphorus 2DES may provide a new venue for exploring exotic many-body physics that are potentially relevant for topological quantum computing.

The combination of high mobility and a variable bandgap makes phosphorene a promising candidate as an electronic and opto-electronic material. In the "more Moore" domain, the FET miniaturization has to be accompanied by faster and more power-efficient operation - the "triple dividends of MOSFET scaling" - in order to maintain continuous performance improvements $[120,5]$. To this end, the role of contact resistance and short channel effects has been investigated in black phosphorus FETs with channel length down to $100 \mathrm{~nm}$ [121]. In the "more than Moore" domain, high speed operation of RF transistors based on few-layer phosphorene has been demonstrated; cutoff frequency $f_{T}$ and maximum oscillation frequency $f_{\max }$ of $12 \mathrm{GHz}$ and $20 \mathrm{GHz}$, respectively, have been achieved in $300 \mathrm{~nm}$ channel length devices (Fig. 5a) [122]. Meanwhile, photo-detectors based on black phosphorus have been shown to work from ultraviolet to mid-infrared region [123-127] with a responsivity up to $\sim 5 \mathrm{~mA} / \mathrm{W}$ at 1.55 um wavelength (ref. 123; Fig. 5b). While these demonstrations are impressive, it is important to note that the research on black phosphorus as an electronic and opto-electronic material is still in an early stage, and there is ample room for future improvements. 


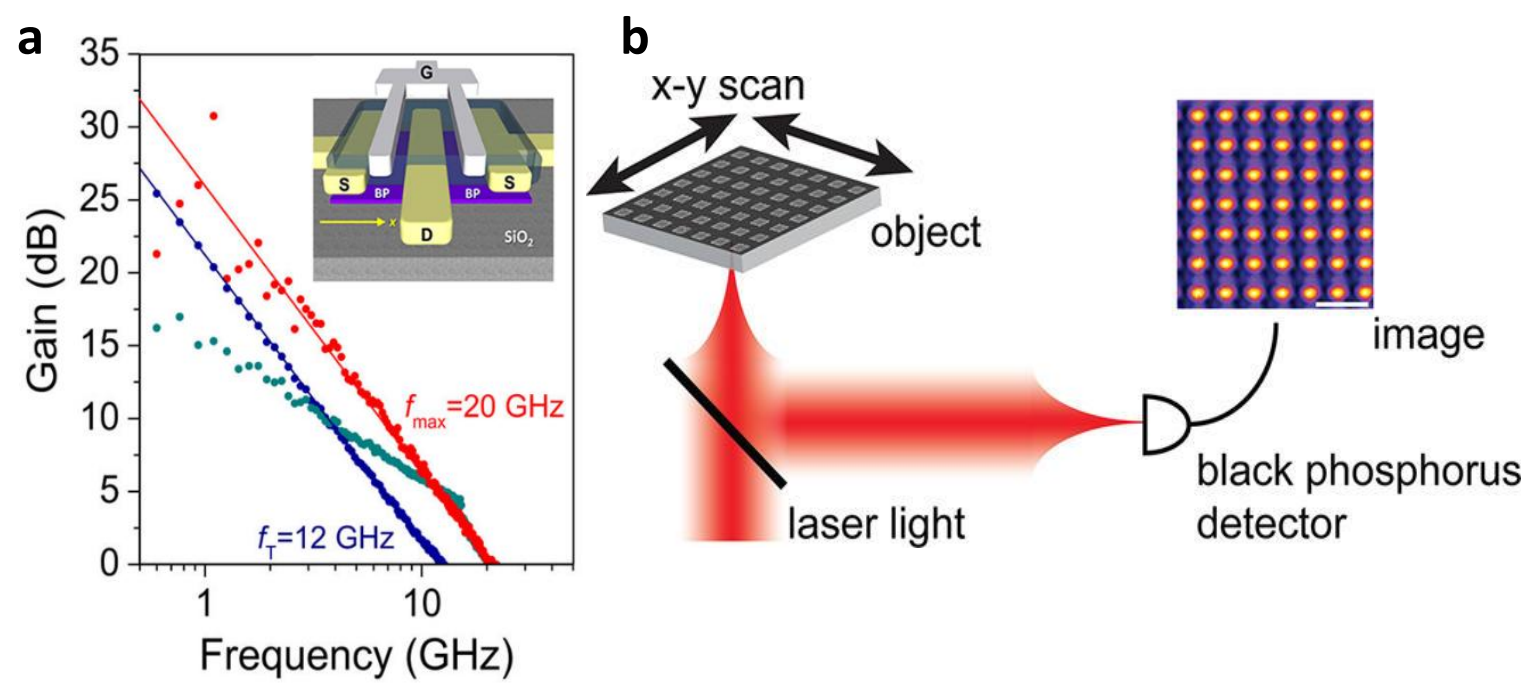

Figure 5. High frequency black phosphorus FET and black phosphorus infrared detector. a, The short-circuit current gain h21 (blue), MSG/MAG (navy), and unilateral power gain (red) of black phosphorus FET with $300 \mathrm{~nm}$ channel length at GHz frequency. The device has $f_{T}=12$ $\mathrm{GHz}$ and $f_{\max }=20 \mathrm{GHz}$ after de-embedding. Inset: schematic of the device structure. Adapted from ref. 122. b, Black phosphorus infrared photo-detector. Shown here is the schematic of the imaging process. Using the black phosphorus device as a point-like detector in a confocal microscope setup, diffraction-limited optical images with submicron resolution can be obtained. Adapted from ref. 123.

\section{Perspectives and Challenges}

The fundamental research and applications of elemental 2D materials is still in its infancy, and the germanene and stanene FETs have not yet been realized. Despite such short history, the progress has been remarkable and there is enormous potential for further improvements and development of new nano-electronic devices. A distinct advantage of 2D materials is their supposed scalability, which is qualitatively captured by the scale length:

$$
\lambda=\sqrt{t_{c h} t_{o x} \varepsilon_{c h} / \varepsilon_{o x}}
$$


where $\varepsilon_{c h}$ and $\varepsilon_{b a r}$ are the dielectric constants of the channel and gate oxide, $t_{c h}$ and $t_{o x}$ are thicknesses of the channel and oxide. $\lambda$ largely determines the limit of the gate length before undesirable short-channel effects set in [128]. Granted everything else equal, the atomically thin 2D channel provides further scaling beyond FET technology based on conventional 3D bulk semiconductors (more elaborate calculations gives a more optimistic estimation of $\lambda$ for atomically thin channels [129]). Elemental 2D materials discussed in this review offer additional advantages: availability of a variable bandgap (in phosphorene), compatibility with existing Si technology (for silicene and germanene), and potential high sample purity as a result of their mono-element nature.

The advantages certainly give elemental 2D materials a head start in the race. But for them to be viable at high operation speed and under extreme scaling, they will have to meet a minimum set of requirements that are summarized in a "wish list" in ref. 5. The wish list outlined the requirements on five aspects of a material's properties: bandgap, effective mass, heat transport, contact resistance, and scale length. Phosphorene is so far the most advanced in terms of FET development among elemental 2D materials besides graphene, and there have been impressive experimental and theoretical efforts addressing the issues related to the wish list $[58,61,65,91,92,94,121,122,130-142]$. But given the short history, the available data are simply too fragmentary to allow for a meaningful comparison with the FET figure of merit from mature technologies according to the wish list. The list, however, is still tremendously useful for identifying some of the key issues that should be the focus of future study. Future challenges include:

(1) Determining the bandgap of few-layer phosphorene as a function of the number of layers. The bandgap is currently inferred from photoluminescence spectroscopy that can be dominated by defect and impurity states rather than the intrinsic bandgap, and different 
studies have reported widely different bandgap values from 1.32 to $1.75 \mathrm{eV}$ for monolayer phosphorene [91,142-144].

(2) Improving the room-temperature carrier mobility, and characterizing carrier velocity under varying electric field strength. This will give a realistic estimate of the operation speed of RF FETs based on few-layer phosphorene.

(3) Reducing the contact resistance down to the order of $0.01 \mathrm{Ohm} \mathrm{mm}$ as specified by the International Technology Roadmap for Semiconductors (ITRS) 2013 edition.

(4) Finding a suitable gate dielectric material that could form clean interface to the channel, protect the channel from degradation in air, and meanwhile withstand aggressive scaling. It has been shown that insulating 2D materials such as $\mathrm{hBN}$ may be a good candidate [112].

(5) Large scale production of elemental 2D materials on an insulating substrate remains a challenge. For phosphorene, this means designing novel chemical routes for largescale growth (instead of mechanical cleaving that are only suitable for small samples used in laboratories; one such attempt has been reported [145]). For silicene and germanene, this means transferring the atomic layer to a desirable insulating substrate without compromising the sample quality, or direct growth on insulating substrates.

The success of elemental 2D materials should not be judged solely based on their application in nanoelectronics. They are very sensitive to external perturbations, which may make them promising candidates for various types of sensor [96,146-150]. The interband optical transitions of few-layer phosphorene cover a wide spectrum range from visible to midinfrared that is important for telecommunication and solar energy harvesting $[132,151,152]$. Together with a high mobility and the capability to further fine-tune the bandgap with mechanical strain and/or electrical field, monolayer and few-layer phosphorene could lead to optoelectronic devices over a broad spectral range. Last but not least, the semi-metallic (group 
IV) and semiconducting (group V) 2D elemental materials could potentially find their applications in flexible, transparent electronics/optoelectronics [146,153,154].

As the material research community turns its attention to $2 \mathrm{D}$ materials beyond graphene, new 2D materials are discovered (or re-discovered) on a weekly basis. There are estimated to be a few hundred more that are waiting to be explored, and the research on $2 \mathrm{D}$ materials will stay active in the foreseeable future. Among the vast number of $2 \mathrm{D}$ materials, elemental 2D materials occupies a special place due to their diverse properties and their mono-elemental nature. It's conceivable that eventually only few 2D materials will find their way into industry, if at all. We believe that elemental 2D materials will be among those selected few.

\section{Acknowledgements}

We acknowledge helpful discussions with S. Cahangirov, L. Xian and Likai Li. Y.Z. acknowledges support from NSF of China (grant nos. 11425415 and 11421404) and National Basic Research Program of China (973 Program; grant no. 2013CB921902). AR acknowledges support from the European Research Council (ERC-2010-AdG-267374, DYNamo), Spanish Grant (FIS2013-46159-C3-1-P), Grupos Consolidados UPV/EHU del Gobierno Vasco (IT578-13), and AFOSR Grant No. FA2386-15-1-0006 AOARD 144088, H2020-NMP-2014. 


\section{References}

[1] Fischetti M V, Fu B and Vandenberghe W G, 2013 IEEE Trans. Electron Devices 60, 3862

[2] Le Lay G, 2D materials: Silicene transistors. 2015 Nat. Nanotechnol. 10, 202

[3] Novoselov K S et al. 2004 Science 306, 666

[4] Ferrari A C et al. Science and technology roadmap for graphene, related two-dimensional crystals, and hybrid systems 2015 Nanoscale 7, 4598

[5]Schwierz F, Pezoldt J and Granzner R, 2015 Nanoscale 7, 8261

[6] Schwierz F, 2010 Nat. Nanotechnol. 5, 487

[7] Georgakilas V et al. 2012 Chem. Rev. 112, 6156

[8] Han M Y, Ozyilmaz B, Zhang Y. and Kim P 2007 Phys. Rev. Lett. 98, 206805

[9] Zhang $Y$ et al. 2009 Nature 459, 820

[10] Lui C H, Li Z, Mak K F, Cappelluti E and Heinz T F 2011 Nat. Phys. 7, 944

[11] Nevius M S et al. 2015 Phys. Rev. Lett. 115, 136802

[12] Miró P, Audiffred M, and Heine, T 2014 Chem. Soc. Rev. 43, 6537

[13] Geim A K and Grigorieva IV 2013 Nature 499, 419

[14] Balendhran S, Walia S, Nili H, Sriram S and Bhaskaran M. 2015 Small 11, 640

[15] Mannix A J et al. 2015 Science 350, 1513

[16] Takeda K and Shiraishi K 1994 Phys. Rev. B 50, 14916

[17] Cahangirov S, Topsakal M, Aktürk E, Şahin H and Ciraci S 2009 Phys. Rev. Lett. 102, 236804

[18] Ezawa M A 2012 New J. Phys. 14, 033003

[19] Xu Y et al. 2013 Phys. Rev. Lett. 111, 136804

[20] Matthes L, Pulci O and Bechstedt F 2013 J. Phys. Condens. Matter 25, 395305

[21] Vogt P et al. 2012 Phys. Rev. Lett. 108, 155501

[22] Dávila M E, Xian L, Cahangirov S, Rubio A and Le Lay, G 2014 New J. Phys. 16, 095002

[23] Derivaz M et al. 2015 Nano Lett. 15, 2510

[24] Zhu F et al. 2015 Nat. Mater. 14, 1020

[25] Li L et al. 2014 Nat. Nanotechnol. 9, 372

[26] Tao L et al. 2015 Nat. Nanotechnol. 10, 227

[27] Nicolosi V, Chhowalla M, Kanatzidis M G, Strano M S and Coleman J N 2013 Science 340, 1226419

[28] Şahin H et al. 2009 Phys. Rev. B 80, 155453

[29] Tang P et al. 2014 Phys. Rev. B 90, 121408(R)

[30] Xu Y, Tang P and Zhang S-C 2015 Phys. Rev. B 92, 081112

[31] Chiappe D. et al. 2014 Adv. Mater. 26, 2096

[32] Tao L et al. 2015 Nat. Nanotechnol. 10, 227

[33] Cahangirov S et al. 2014 Phys. Rev. B 90, 035448

[34] Fu H et al. 2015 Nanoscale 7, 15880

[35] Shirai T et al. 2014 Phys. Rev. B 89, 241403

[36] Boustani I, Quandt A, Hernández E and Rubio A 1999 J. Chem. Phys. 110, 3176

[37] Tang $\mathrm{H}$ and Ismail-Beigi S 2007 Phys. Rev. Lett. 99, 115501

[38] De Padova P et al. 2013 J. Phys. Condens. Matter 25, 382202

[39] Chen L et al. 2012 Phys. Rev. Lett. 109, 056804

[40] Cahangirov Set al. 2013 Phys. Rev. B 88, 035432

[41] Liu C-C, Feng W and Yao Y 2011 Phys. Rev. Lett. 107, 076802

[42] Ezawa M 2015 J. Phys. Soc. Jpn. 84, 121003

[43] Ezawa M 2014 J. Supercond. Nov. Magn. 28, 1249

[44] Jia T-T et al. 2015 J. Phys. Chem. C 119, 20747

[45] Tabert C J, Carbotte J P and Nicol E J 2015 Phys. Rev. B 91, 035423

[46] Zhang L-D, Yang F and Yao Y 2015 Sci. Rep. 5, 8203

[47] Zhang L-D, Yang F and Yao Y 2015 Phys. Rev. B 92, 104504

[48] Elias D C et al. 2009 Science 323, 610 
[49] Cudazzo P, Attaccalite C, Tokatly I . and Rubio A 2010 Phys. Rev. Lett. 104, 226804

[50] Zólyomi V, Wallbank J R and Fal'ko, V I 2014 2D Mater. 1, 011005

[51] Yu W-Z, Yan J-A and Gao S-P 2015 Nanoscale Res. Lett. 10, 351

[52] Lin S-Y, Chang S-L, Tran N T T, Yang P-H and Lin M-F 2015 Phys. Chem. Chem. Phys. 17, 26443

[53] Takahashi T, Tokailin H, Suzuki S, Sagawa T and Shirotani I 1985 J. Phys. C Solid State Phys. 18, 825

[54] Asahina H and Morita A 1984 J. Phys. C Solid State Phys. 17, 1839

[55] Morita A 1986 Appl. Phys. Mater. Sci. Process. 39, 227

[56] Takao Y, Asahina H and Morita A 1981 J. Phys. Soc. Jpn. 50, 3362

[57] Asahina H, Shindo K and Morita A 1982 J. Phys. Soc. Jpn. 51, 1193

[58] Tran V and Yang L 2014 Phys. Rev. B 89, 245407

[59] Tran V, Soklaski R, Liang Y and Yang L 2014 Phys. Rev. B 89, 235319

[60] Rudenko A N and Katsnelson M I 2014 Phys. Rev. B 89, 201408(R)

[61] Das S et al. 2014 Nano Lett. 14, 5733

[62] Low T et al. 2014 Phys. Rev. B 90, 075434

[63] Liang L et al. 2014 Nano Lett. 14, 6400

[64] Akahama Y, Endo S and Narita S 1983 J. Phys. Soc. Jpn. 52, 2148

[65] Qiao J, Kong X, Hu Z-X, Yang F and Ji W 2014 Nat. Commun. 5, 4475

[66] Low T et al. 2014 Phys. Rev. Lett. 113, 106802

[67] Liu Q, Zhang X, Abdalla L B, Fazzio A and Zunger A 2015 Nano Lett. 15, 1222

[68] Kim J et al. 2015 Science 349, 723

[69] Cartz L, Srinivasa S R, Riedner R J, Jorgensen J D and Worlton T G 1979 J. Chem. Phys. 71, 1718 (1979)

[70] Shirotani I et al. 1990 Phys. Lett. A 144, 102

[71] Akahama Y and Endo S 1997 Solid State Commun. 104, 307

[72] Gong, P-L et al. 2016 Phys. Rev. B 93, 195434

[73] Fei R and Yang L 2014 Nano Lett. 14, 2884

[74] Rodin A S, Carvalho A and Castro Neto A H 2014 Phys. Rev. Lett. 112, 176801

[75] Xiang Z J et al. 2015 Phys. Rev. Lett. 115, 186403

[76] Keyes R W 1953 Phys. Rev. 92, 580

[77] Okajima M, Endo S, Akahama Y and Narita S 1984 Jpn. J. Appl. Phys. 23, 15

[78] Lin C-L et al. 2012 Appl. Phys. Express 5, 045802

[79] Fukaya Y et al. 2013 Phys. Rev. B 88, 205413

[80] Kawahara K et al. 2014 Surf. Sci. 623, 25-28

[81] Resta A et al. 2013 Sci. Rep. 3, 2399

[82] Liu Z-L et al. 2014 New J. Phys. 16, 075006

[83] Rahman M S, Nakagawa T and Mizuno S 2015 Jpn. J. Appl. Phys. 54, 015502

[84] Fleurence A et al. 2012 Phys. Rev. Lett. 108, 245501

[85] Meng L et al. 2013 Nano Lett. 13, 685

[86] Lin C-L et al. 2013 Phys. Rev. Lett. 110, 076801

[87] Stephan R, Hanf M-C and Sonnet P 2015 J. Phys. Condens. Matter 27, 015002

[88] Guo Z-X, Furuya S, Iwata J and Oshiyama A 2013 Phys. Rev. B 87, 235435

[89] Li L et al. 2014 Adv. Mater. 26, 4820

[90] Švec M et al. 2014 Phys. Rev. B 89, 201412

[91] Liu H et al. 2014. ACS Nano 8, 4033-4041 (2014).

[92] Koenig S P, Doganov R A, Schmidt H, Neto A H C and Özyilmaz B 2014 Appl. Phys. Lett. 104, 103106

[93] Castellanos-Gomez A et al. 2014 2D Mater. 1, 025001

[94] Xia F, Wang H and Jia Y 2014 Nat. Commun. 5, 4458

[95] Kang J. et al. 2015 ACS Nano 9, 3596

[96] Hanlon D et al. 2015 Nature Comm. 6, 8563

[97] Yasaei P et al. 2015 Adv. Mater. 27, 1887 
[98] Zandvliet H J W 2014 Nano Today 9, 691-694

[99] Li L et al. 2013 http://meetings.aps.org.lama.univ-amu.fr/link/BAPS.2013.MAR.J6.13

[100] Churchill H O and Jarillo-Herrero P 2014 Nat. Nanotechnol. 9, 330

[101] Li L et al. 2015 Nat. Nanotechnol. 10, 608

[102] Favron A et al. 2015 Nat. Mater. 14, 826

[103] Wood J. D. et al. 2014 Nano Lett. 14, 6964

[104] Kim J-S et al. 2015 Sci. Rep. 5, 8989

[105] Wang Z, Islam A, Yang R, Zheng X and Feng P X-L 2015 J. Vac. Sci. Technol. B 33, 052202

[106] Ando T, Fowler A B and Stern F 1982 Rev. Mod. Phys. 54, 437

[107] Tayari V et al. 2015. Nat. Commun. 6, 7702

[108] Gillgren N et al. 2015 2D Mater. 2, 011001

[109] Chen X et al. 2015 Nat. Commun. 6, 6088

[110] Pfeiffer L and West K W 2003 Phys. E 20, 57

[111] Dean C R et al. 2010 Nat Nano 5, 722

[112] Meric let al. 2013 Proc. IEEE 101, 1609

[113] Ponomarenko L A et al. 2011 Nat. Phys. 7, 958

[114] Hunt B et al. 2013 Science 340, 1427

[115] Young A F et al. 2013 Nature 505, 528

[116] Maher P et al. 2014 Science 345, 61

[117] Li L et al. 2016 Nature Nano 11, 593

[118] Akahama Y, Endo S and Narita S 1983 J. Phys. Soc. Jpn. 52, 2148

[119] Falson J et al. 2015 Nat. Phys. 11, 347

[120] del Alamo J A 2011 Nature 479, 317

[121] Du Y, Liu H, Deng Y and Ye P D 2014 ACS Nano 8, 10035

[122] Wang $\mathrm{H}$ et al. 2014 Nano Lett. 14, 6424

[123] Engel M, Steiner M and Avouris P 2014 Nano Lett. 14, 6414

[124] Buscema M et al. 2014 Nano Lett. 14, 3347

[125] Hong T et al. 2014 Nanoscale 6, 8978

[126] Wu J et al. 2015 ACS Nano 9, 8070

[127] Yuan H et al. 2015 Nat. Nanotechnol. 10, 707

[128] Yan R-., Ourmazd A and Lee K F 1992 Electron Devices IEEE Trans. On 39, 1704

[129] Zhang Q, Lu Y, Xing H G, Koester S J and Koswatta S O 2010 IEEE Electron Device Lett. 31, 531

[130] Li L et al. Nat. Nanotechnol. 9, 372

[131] Qiao J, Kong X, Hu Z-X, Yang F and Ji W 2014 arxiv.org/vc/arxiv/papers/1401/1401.5045v1.pdf

[132] Deng $Y$ et al. 2014 ACS Nano 8, 8292

[133] Tran V and Yang L 2014 Phys. Rev. B 89, 245407

[134] Favron A et al. 2015 Nature Mat. 14, 826

[135] Han X, Morgan Stewart H, Shevlin S A, Catlow C R A and Guo Z X 2014 Nano Lett. 14, 4607

[136] Gong K, Zhang L, Ji W and Guo H 2014 Phys. Rev. B 90, 125441

[137] Doganov R A et al. 2015 Appl. Phys. Lett. 106, 083505

[138] Cao Y et al. 2015 Nano Lett. 15, 4914

[139] Jiang J-W 2015 Nanotechnology 26, 055701

[140] Cao X and Guo J 2015 IEEE Trans. Electron Devices 62, 659

[141] Liu F, Wang Y, Liu X, Wang J and Guo H 2014 IEEE Trans. Electron Devices 61, 3871

[142] Das $S$ et al. 2015 IEEE Electron Device Lett. 36, 621

[143] Xu R et al. 2016 ACS Nano 10, 2046

[144] Yang J et al. 2015 Light Sci. Appl. 4, e312

[145] Wang X et al. 2015 Nat. Nanotechnol. 10, 517

[146] Li X et al. 2015 2D Mater. 2, 031002

[147] Kou L, Frauenheim T and Chen C 2014 J. Phys. Chem. Lett. 5, 2675

[148] Li Y, Yang S and Li J 2014 J. Phys. Chem. C 118, 23970

[149] Peng X, Wei Q and Copple A 2014 Phys. Rev. B 90, 085402 
[150] Manjanath A, Samanta A, Pandey T and Singh A K 2015 Nanotechnology 26, 075701

[151] Dai J and Zeng X C 2014 J. Phys. Chem. Lett. 5, 1289

[152] Ganesan V D S, Zhang C, Feng Y P and Shen L 2015 ArXiv:1507.07343

[153] Zhu W et al. 2015 Nano Lett. 15, 1883

[154] Wei Q and Peng X 2014 Appl. Phys. Lett. 104, 251915 